\title{
Antibiotic-impregnated articulating cement spacer maintained for 7 years in situ for two-stage primary total knee arthroplasty: a case report
}

Yong-Beom Park' ${ }^{1}$ Chul-Won $\mathrm{Ha}^{2,3,4^{*}}$, Jae Won Jang ${ }^{5}$ and Manyoung Kim ${ }^{6}$

\begin{abstract}
Background: Antibiotic-impregnated articulating cement spacers can maintain interim joint motion with the potential to enhance functional status and improve patient satisfaction. Articular surfaces with cement against cement have raised concerns regarding mechanical complications and cement debris during knee motion. However, long-term clinical conditions regarding these concerns are not well addressed.

Case presentation: We report a case in which articulating cement spacers were maintained in situ for 7 years. The patient had severe left knee pain with an ankylosing knee and severe tricompartmental arthritis due to tuberculous infection. We planned to perform one- or two-stage primary total knee arthroplasty (TKA), depending on the presence of infection. Persistent osteomyelitis was found intraoperatively. The second-stage TKA was delayed on the patient's request. As the patient was satisfied with the improved knee function and pain relief after using articulating cement spacers. No symptom or sign that suggested recurrent infection or systemic toxicity was found during the 7-year follow-up. However, it seemed that the bone loss progressed insidiously. At the 7-year follow-up, a broken articulating cement spacer and medial femoral condylar fracture were found. The second-stage TKA was performed, and a considerable amount of bone loss surrounded by dense granulation tissue was observed intraoperatively. Excisional biopsy of the tissue revealed chronic foreign body reaction with infiltration of giant cells and macrophages.
\end{abstract}

Conclusion: Although the articular spacers were maintained for 7 years without major complications, regular observation of the development and progress of bone loss was required. Surgeons should take considerable bone loss into account during conversion TKA in patients with a prolonged retention of articulating cement spacers.

Keywords: Total knee arthroplasty, Infection, Articulating spacer, Retention

\section{Background}

The usefulness of antibiotic-impregnated articulating cement spacers has been extensively studied for the treatment of infections after two-stage revision total knee arthroplasty (TKA) [1-13]. It is also used in two-stage primary TKA to treat advanced knee arthritis with

\footnotetext{
*Correspondence: chulwon.ha@gmail.com; hacw@skku.edu

${ }^{2}$ Department of Orthopaedic Surgery, Samsung Medical Center,

Sungkyunkwan University School of Medicine, 81 Irwon-ro, Gangnam-gu, Seoul 06351, South Korea

${ }^{3}$ Stem Cell and Regenerative Medicine Institute, Samsung Medical Center, 81 Irwon-ro, Gangnam-gu, Seoul 06351, South Korea

Full list of author information is available at the end of the article
}

coexistent joint infection [14]. Static spacers can lead to prolonged restriction of knee motion with limited knee function $[4,15,16]$. However, articulating spacers can be designed to allow range of motion and protect weight bearing between stages $[1,3,11]$. Compared with static spacers, articulating spacers have shown greater range of motion at final follow-up [2, 4, 5, 8-10], less bone loss between stages $[2,4,8,9]$, and decreased difficulty in exposure during reimplantation $[2,8-10,12]$. In addition, the currently available evidence suggests that articulating spacers have similar or better outcomes than static spacers in infection control $[2,5,7-10,17]$.

(c) The Author(s). 2019 Open Access This article is distributed under the terms of the Creative Commons Attribution 4.0 International License (http://creativecommons.org/licenses/by/4.0/), which permits unrestricted use, distribution, and reproduction in any medium, provided you give appropriate credit to the original author(s) and the source, provide a link to the Creative Commons license, and indicate if changes were made. The Creative Commons Public Domain Dedication waiver (http://creativecommons.org/publicdomain/zero/1.0/) applies to the data made available in this article, unless otherwise stated. 
Articulating spacers have gained popularity owing to these advantages. However, few studies have reported retention of articulating spacers in infected TKA [5, 18, 19]. In addition, detailed clinical information on long-term follow-up of retained articulating cement spacers is limited. Articular surfaces with cement against cement cause concern with regard to mechanical complications and cement debris during knee motion in long-term clinical conditions. Here, we report a case of in situ maintenance of articulating cement spacers during the interim period of two-stage primary TKA for 7 years. We adhere to CARE (CAse REport) Statement and Checklist for accurate reporting [20].

\section{Case presentation}

A 44-year-old man was admitted to our clinic because of left knee pain. He had pulmonary tuberculosis at 6 years old. He presented pus-forming arthritis, which was presumably tuberculous arthritis, in the left knee after 2 years, with spontaneous remission after closure of the draining sinuses. Thereafter, no recurrent symptom of infection was observed. However, the deformity and growth disturbance progressed with the knee pain. $\mathrm{He}$ had limb lengthening and alignment correction for the leg length discrepancy and genu valgum. However, his left knee pain continued despite the deformity correction. Radiographs showed a fused knee with severe tricompartmental arthritis (Fig. 1). Severe limitation in range of motion was observed on the left knee. We planned to perform one- or two-stage primary TKA depending on the presence of infection [14].
Intraoperatively, a large subchondral abscess was found in the lateral femoral condyle and lateral tibial plateau after takedown of the fusion (Fig. 1d). On the basis of the necrotizing inflammation with granuloma in the frozen-section biopsy, active tuberculosis was suspected. Aggressive debridement and curettage of the infected and necrotic bone and soft tissues were performed.. After bone cuts and soft tissue balancing to prepare for TKA, articulating cement spacers (vancomycin $4 \mathrm{~g}$ and streptomycin $2 \mathrm{~g}$ per 1 batch) were made intraoperatively and applied to the tibial and femoral sides in sequence using intraoperative cement molds with a previously described technique $[14,21]$ (Fig. 2). Relative medial and lateral stabilities were confirmed intraoperatively after inserting the articulating cement spacers. The diagnosis of tuberculosis infection was confirmed by isolating Mycobacterium tuberculosis from cultures. We decided to delay the TKA for at least 6 to 9 months to allow the administration of antituberculous drugs [22]. Evaluation at 1-year follow-up revealed no recurrent infection after sufficient antituberculous drug treatment. Therefore, we recommended TKA surgery as planned. However, the patient was comfortable with the articulating cement spacers. He refused a conversion to TKA for personal reasons. At every visit thereafter, he consistently wanted to delay the second-stage surgery.

Painless activities were possible, including gait, step ascent and descent, and rising from a chair with full load bearing. No evidence was found that suggested recurrent infection or systemic toxicity. Laboratory markers of infection, and renal and hepatic toxicities were followed up once in a year. No abnormal finding was identified. A

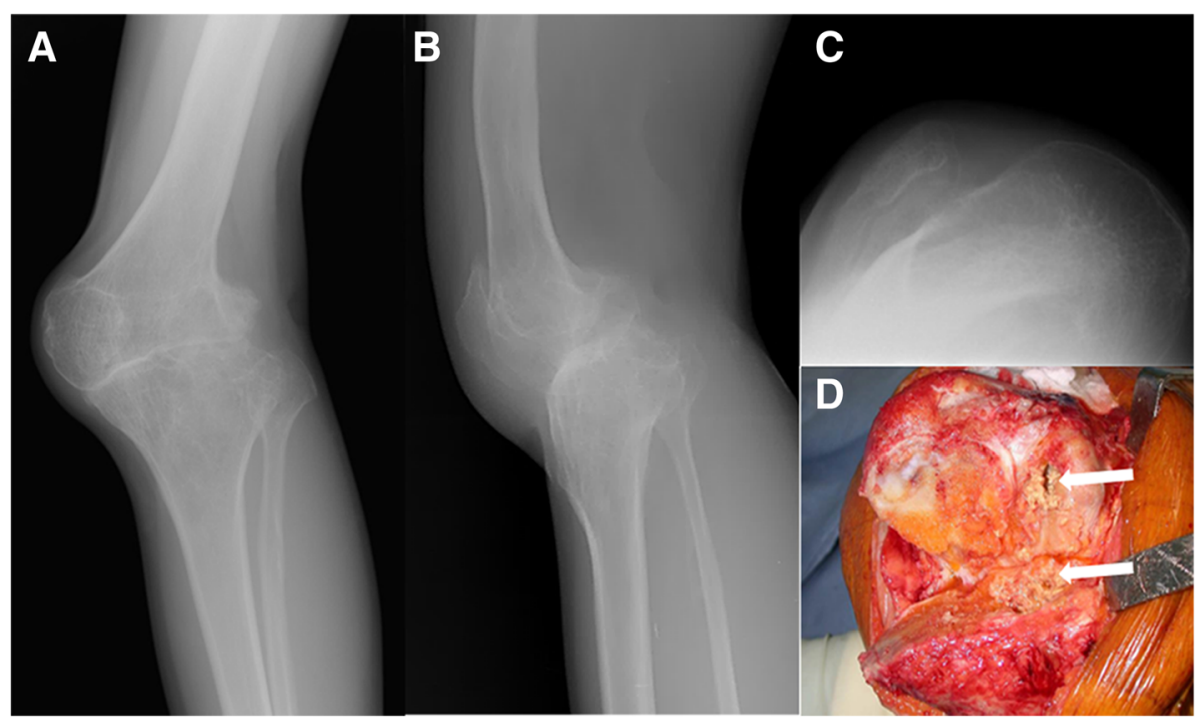

Fig. 1 a-d Preoperative knee radiographs in the anteroposterior (a), lateral (b), and merchant views (c), showing tibiofemoral ankylosis with deformity and tricompartmental arthritis. (d) An intraoperative photograph taken after takedown of fusion, showing necrotic bone (arrow) in the lateral femoral condyle and lateral tibial plateau 


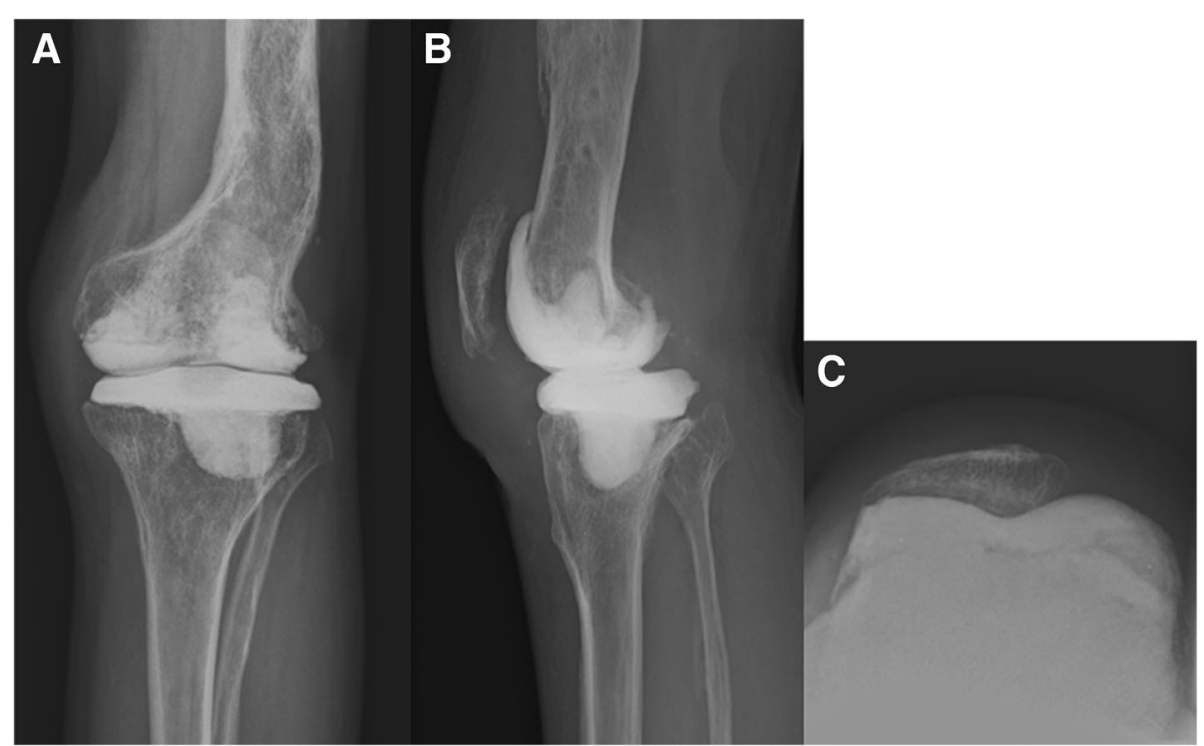

Fig. 2 a-c Postoperative anteroposterior (a), lateral (b), and merchant knee radiographs (c) taken at 6 weeks after the implantation of articulating spacers. The medullary protruded portion of the tibial articulating spacer was deviated to the lateral side because of the contained bone defect of the lateral tibial plateau after debridement of the infected bone. The contained bone defect was also left in the lateral femoral condyle after debridement of the infected bone

slowly progressing small bone loss was observed at the bone-cement interface. However, no notable mechanical problem such as subluxation, dislocation, periprosthetic, or implant fracture were observed on the serial knee radiographs (Fig. 3a and b). At the 7-year follow-up, the patient complained of left knee pain for 2 months. Radiographic and computed tomographic evaluations revealed collapse of the medial femoral condyle with fracture of the femoral articulating spacer component (Figs. 3c and 4).
At 7 years after the implantation of spacers, we converted the articulating spacers to TKA using the NexGen Legacy Constrained Condylar Knee system (Zimmer, Warsaw, IN, USA). The appearance of the knee joint and the finding from the intraoperative frozen-section examination suggested no residual infection. The femoral component of the cement spacer, along with the imaging study, showed a bisecting vertical fracture (Fig. 5a). The tibial component was intact (Fig. 5b). After removing the cement spacers, the dense

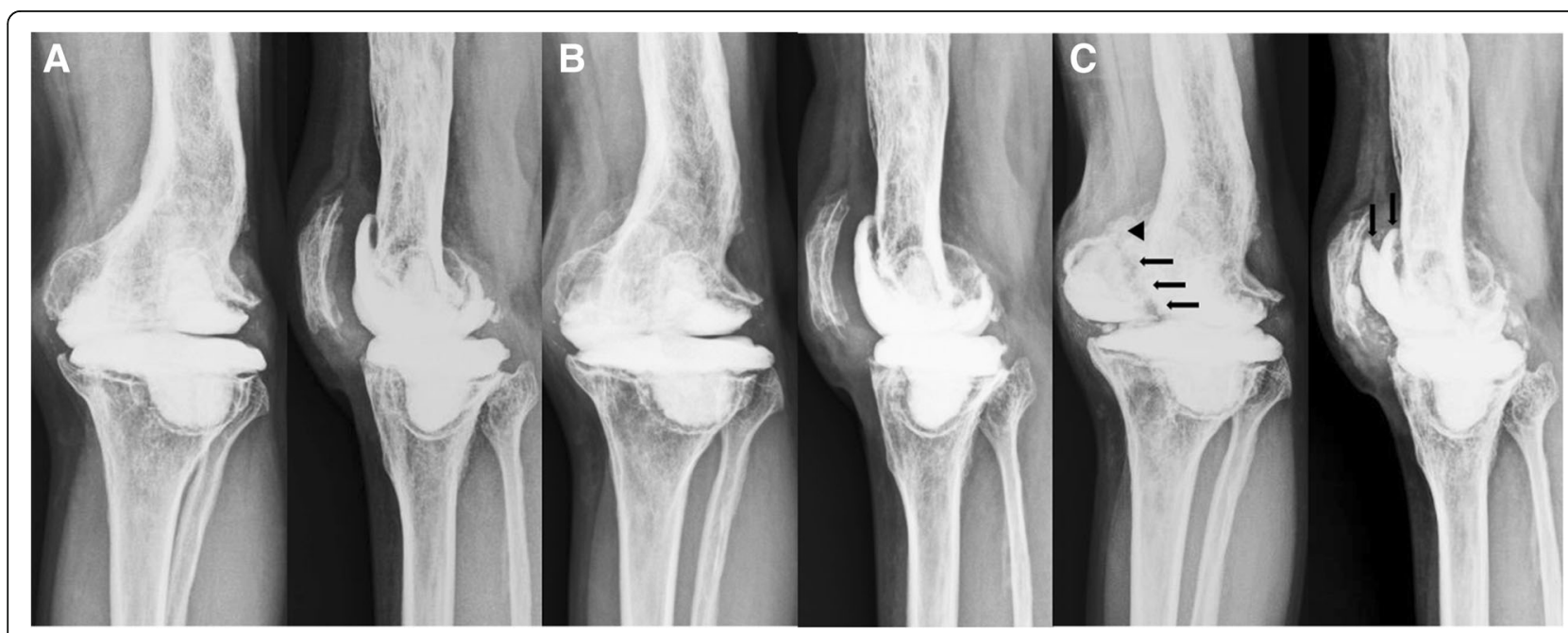

Fig. 3 a-c Postoperative anteroposterior and lateral knee radiographs taken at 5 years (a), 6 years (b), and 7 years (c) after the implantation of articulating spacers. (a, b) Radiolucent lines along the bone-cement interface. (b) The longitudinal split fracture of the femoral articulating spacer component (arrow) with cortical breakage of the medial femoral condyle (arrowhead) observed at 7 years after the implantation of articulating spacers 


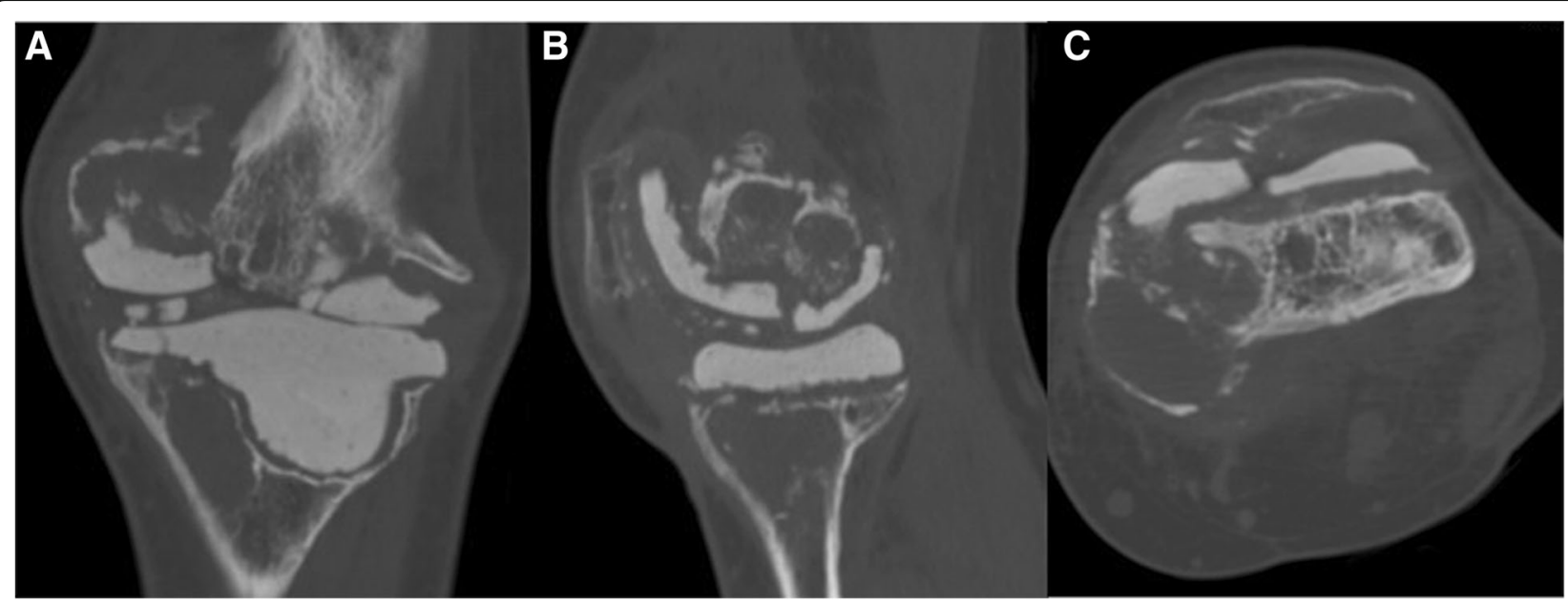

Fig. 4 a-c Left knee computed tomography scans of the coronal (a), medial sagittal (b), and femoral axial planes (c) at 7 years after the implantation of articulating spacers, showing the underlying bone loss around the femoral and tibial components of the articulating spacers. It was more severe in the medial femoral condyle and medial tibial plateau. Cortical thinning and discontinuity of the medial femoral condyle could be observed

fibrotic granulation tissue surrounding the bone surface was exposed, especially in the medial femoral condyle (Fig. 5b). Excisional biopsy of the tissues was performed. Considerable bone loss with cortical bone breakage was observed in the medial femoral condyle after the debridement of the granulation tissue and necrotic bone (Fig. 5c). We conducted wiring for the cortical bone breakage, and an allogenous morselized bone graft was performed for the bone loss. Augmentation blocks were additionally used for the remnant bone defect. In the proximal tibia, the considerably contained bone defect was restored with an allogenous morselized bone graft (Fig. 6a). Histological examination of the specimens from the bone surface of the medial femoral condyle showed marked fibrosis with a significant foreign body reaction characterized by infiltration of giant cells and macrophages (Fig. 7). Direct visualization of polymethyl methacrylate (PMMA) particles was impossible because PMMA in the bone cement was dissolved in the organic solvent used during the routine histological processing. However, a negative pattern of the surrounding foreign body giant cells was observed, with various sizes $[23$, 24]. Many tiny particles were assumed to be abraded zirconium dioxide, an X-ray contrast medium used as an ingredient in the Palacos $\mathrm{R}$ bone-cement [25]. These particles were mainly located within the cytoplasm of

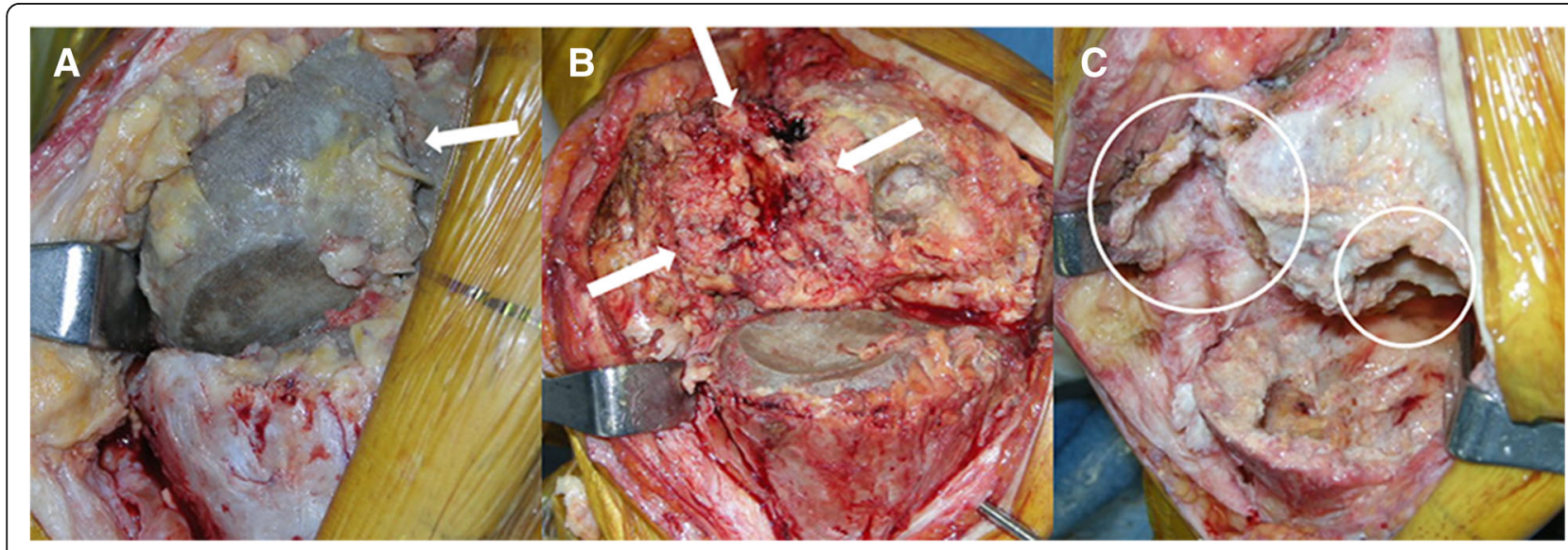

Fig. 5 a-c (a) Longitudinal split fracture of the femoral component of the articulating spacer (arrow) found intraoperatively. (b) After removal of the femoral spacer component, a dense layer of fibrous tissue surrounding the femoral bony surface was found, particularly in the medial femoral condyle (white arrow). The tibial component of the articulating spacer was intact. (c) After meticulous debridement of the soft tissue and bone, considerable amounts of bone loss can be found in the medial femoral condyle (large circle) as compared with the lateral femoral condyle (small circle) 


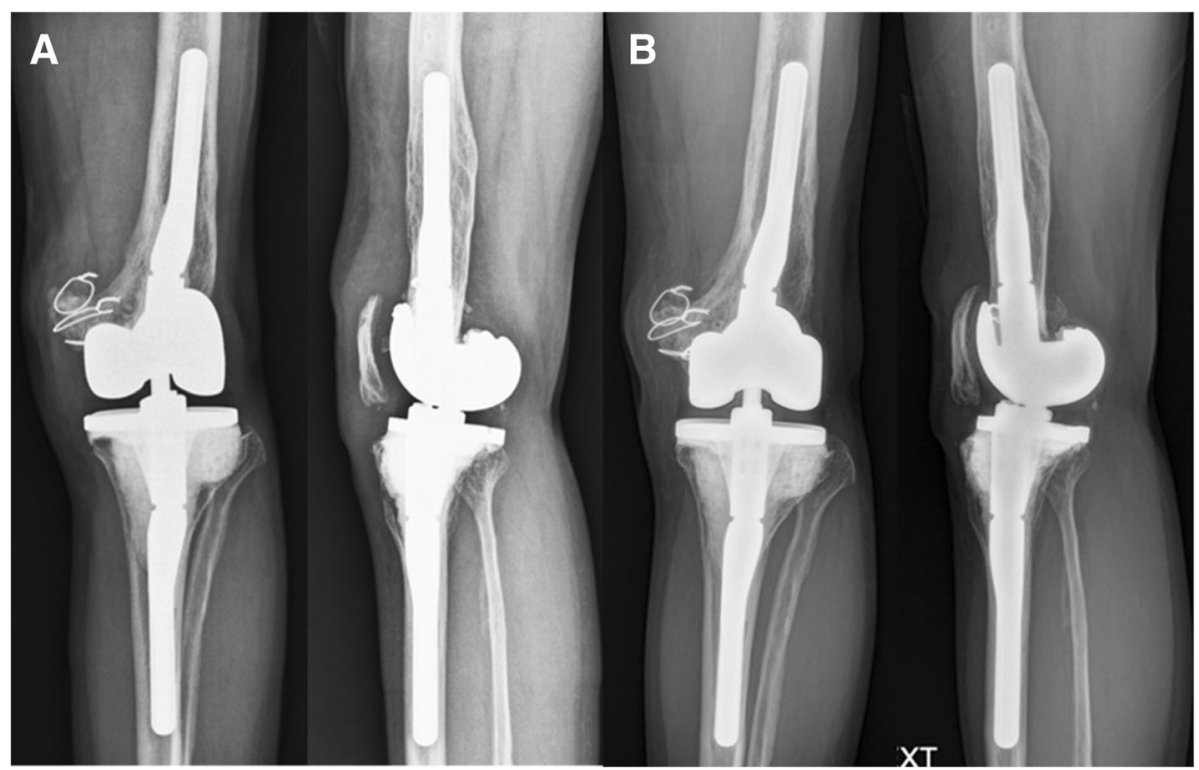

Fig. $\mathbf{6}$ a, b Postoperative anteroposterior and lateral knee radiographs taken at 6 weeks (a) and 2 years (b) after the second-stage surgery

mononuclear macrophages. No evidence of infection or malignant lesion was found.

At the 2-year follow-up, the patient had a passive range of motion from $0^{\circ}$ to $90^{\circ}$, with stability. TKA was maintained without osteolysis (Fig. 6b). He was satisfied with the clinical outcome after TKA conversion.

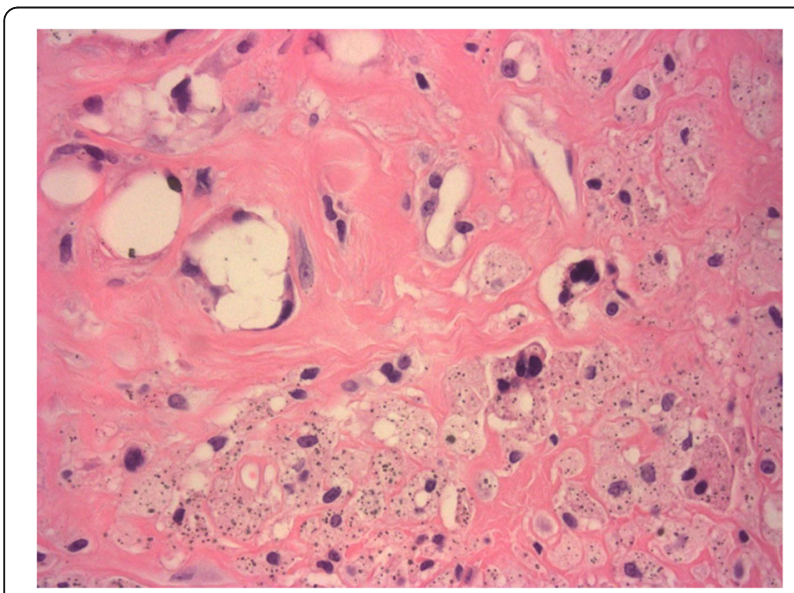

Fig. 7 Photomicrograph of foreign body granulation tissue showing variable sizes of negative patterns of polymethyl methacrylate (PMMA) particles surrounded by giant cells and macrophages. PMMA particles are well known to dissolve during normal preparation for paraffin embedding. Tiny particles were assumed to be abraded X-ray contrast medium used as an ingredient in bone cement. They were accumulated in phagocytes with sizes ranging from 0.5 to $1 \mu \mathrm{m}$ in diameter. Marked fibrous tissue surrounding the inflammatory cells could be observed (hematoxylin and eosin staining; original magnification, $\times 400)$

\section{Discussion and conclusions}

This paper reports a case of in situ maintenance of articulating cement spacers for approximately 7 years. Only a few studies have reported retention of cement spacers for TKA-related infection $[5,18,19]$. Two studies used re-sterilized prosthesis spacers to treat the infection $[5,18]$. The presence of a metallic or plastic component could favor bacterial adhesion [26]. In addition, articulating cement spacers could contain a relatively larger amount of antibiotic power than re-sterilized prosthesis spacers. Although one study has used cement spacers, detailed clinical information regarding the retention of cement spacers has not been documented [19]. Therefore, this is the first case report that investigated the detailed clinical outcome of a patient with retained articulating cement spacers. In this case, no specific functional problem, reinfection, systemic toxicity, or malignant lesion were observed during a long period between the stages. However, the underlying periprosthetic bone loss observed in the second-stage surgery, especially in the medial femoral condyle, seemed to have progressed insidiously, possibly causing the main problem of the long-term maintenance of articulating cement spacers in this case [27]. However, evidence of bone loss was not definite on the serial radiographs except at the bone-cement interface. Plain radiographs tend to underestimate the presence and extent of bone resorption [28, 29]. Several possible reasons can explain bone loss. First, cement spacers themselves could be the cause of periprosthetic bone loss due to micromotion. The amount of bone loss has been reported to be associated with the length of time after 
implantation of cement spacers [9]. In this case, the radiolucent lines along the bone-cement junction implied micromotion of the articulating spacers, although no remarkable evidence of migration was found in the serial knee radiographs. Second, progressive medial or lateral laxity could be a possible cause of bone loss through uneven stress distribution, which could increase the compressive load to the medial side of cement spacers and underlying cancellous bone. The load concentration on the medial compartment might be relevant to the bone loss concentrated on the medial femoral condyle and the feature of the longitudinal split fracture in the femoral component of the cement spacer. However, a considerable amount of bone loss could not be fully explained by these mechanical factors. Foreign-body reaction induced by cement debris might be a possible cause of severe bone loss. In this case, the dense layer of fibrotic connective tissue at the bone-cement interface revealed chronic foreign-body reaction, with phagocytes accumulating tiny particles assumed to be abraded zirconium dioxide. Bone resorption has been reported to be associated with foreign-body reaction induced by the radiopaque agent of bone cement [30,31]. Abraded cement debris can be produced at cement-against-cement articulation [25, 32]. Long-term maintenance of articulating cement spacers could be problematic because of the accumulation of tiny particles. Therefore, in cases of delayed second-stage surgery, regular and precise observation about the development and progress of bone loss is needed. Follow-up with computed tomography could aid the diagnosis and measurement of bone loss to assess the risk of mechanical complications and level of difficulty of the second-stage surgery.

Several factors might have caused the long-term survival of the articulating cement spacers in this case despite that the patient was allowed to perform tolerable load-bearing tasks. First, the patient did not have coronal instability or uncontained bone defects after the first-stage surgery. Motions with articulating cement spacers are suggested to be unsuitable for patients with severe bone loss, ligamentous instability, or extensor mechanism problem [6, 33]. Second, this patient had mild weakness of the ipsilateral lower limb. Therefore, high-level physical activity could be limited. Third, our intraoperative construction technique for articulating cement spacers enables inter-digitation between the irregular bone surface and the cement spacer with an adjustable extension and flexion gap [14, 21]. We applied an intraoperatively manufactured mold to a bolus of bone cement that was put on the irregular bony surface of each femoral and tibial articular sides in the late doughy phase. After a tibial cement spacer was constructed, the thickness of the femoral cement spacer was adjusted to make ideal extension and flexion gaps with the tibial cement spacer. During recurrent knee extension and flexion, the deformed surface of the femoral cement spacer during knee motion was restored by reapplying the mold. Intraoperatively, a high degree of stability was possible in the interface between the bone and the articulating cement spacer or between the femoral and tibial components of the articulating cement spacer, although some lateral laxity unavoidably progressed slowly thereafter.

In this case, the patient had maintained the articular spacers in the left knee in situ for approximately 7 years without major functional problems, reinfection, systemic toxicity, or mechanical complications, despite allowing tolerable load-bearing tasks. The bone loss progressed insidiously, which might have caused difficulties in the second-stage surgery, with probable risk in the development of mechanical problems. Therefore, regular and precise observation about the development and progress of bone loss is required with radiographsor computed tomography in doubtable case. Care should be taken regarding a considerable amount of bone loss in conversion TKA in patients with prolonged retention of articulating cement spacers.

\section{Abbreviations}

PMMA: Polymethyl methacrylate; TKA: Total knee arthroplasty

\section{Acknowledgments}

We thank Tai-Hee Seo BS for her help in the data collection and database management.

\section{Funding}

This research was supported by a grant from the Korea Health Technology R\&D Project through the Korea Health Industry Development Institute (KHIDI), funded by the Ministry of Health and Welfare, Republic of Korea (HI14C3484). The funding sources were not involved in the study design; collection, analysis, or interpretation of the data; writing of the manuscript; or in the decision to submit the manuscript for publication.

\section{Availability of data and materials}

The datasets generated and/or analyzed during the current study are not publicly available due to protection patients` information but are available from the corresponding author on reasonable request.

\section{Authors' contributions}

YBP wrote the manuscript and performed the data collection and interpretation. CWH designed and performed the study, and wrote the manuscript. JWJ performed the literature search, data collection, made figures, and helped write the manuscript. MK performed the literature search and data interpretation, and helped to write the manuscript. All authors read and approved the final manuscript.

\section{Ethics approval and consent to participate}

All procedures performed in studies involving human participants were in accordance with the ethical standards of the institutional and/or national research committee and with the 1964 Helsinki declaration and its later amendments or comparable ethical standards.

\section{Consent for publication}

Written informed consent was obtained from the patient for the publication of this case report and any accompanying images. 


\section{Competing interests}

The authors have no conflict of interest to declare.

\section{Publisher's Note}

Springer Nature remains neutral with regard to jurisdictional claims in published maps and institutional affiliations.

\section{Author details}

'Department of Orthopedic Surgery, Chung-Ang University Hospital, Chung-Ang University College of Medicine, 102 Heukseok-ro, Dongjak-gu, Seoul 06973, South Korea. ²Department of Orthopaedic Surgery, Samsung Medical Center, Sungkyunkwan University School of Medicine, 81 Irwon-ro, Gangnam-gu, Seoul 06351, South Korea. ${ }^{3}$ Stem Cell and Regenerative Medicine Institute, Samsung Medical Center, 81 Irwon-ro, Gangnam-gu, Seoul 06351, South Korea. ${ }^{4}$ Department of Health Sciences and Technology, SAIHST, Sungkyunkwan University, 81 Irwon-ro, Gangnam-gu, Seoul 06351, South Korea. ${ }^{5}$ Department of Orthopedic Surgery, Yonsei Knee and Spine Hospital, 568 Cheonho-daero, Gwangjin-gu, Seoul, South Korea. ${ }^{6}$ Department of Orthopedic Surgery, The Leon Wiltse Memorial Hospital, 560 Gyeongsu-daero, Dongan-gu, Anyang-si, Gyeonggi-do 14112, South Korea.

\section{Received: 25 December 2018 Accepted: 12 April 2019}

\section{Published online: 25 April 2019}

\section{References}

1. Hofmann AA, Kane KR, Tkach TK, Plaster RL, Camargo MP. Treatment of infected total knee arthroplasty using an articulating spacer. Clin Orthop Relat Res. 1995:45-54

2. Fehring TK, Odum S, Calton TF, Mason JB. Articulating versus static spacers in revision total knee arthroplasty for sepsis. The Ranawat Award. Clin Orthop Relat Res. 2000:9-16.

3. Haddad FS, Masri BA, Campbell D, McGraw RW, Beauchamp CP, Duncan CP. The PROSTALAC functional spacer in two-stage revision for infected knee replacements. Prosthesis of antibiotic-loaded acrylic cement. J Bone Joint Surg Br. 2000;82:807-12.

4. Emerson RH Jr, Muncie M, Tarbox TR, Higgins LL. Comparison of a static with a mobile spacer in total knee infection. Clin Orthop Relat Res. 2002: 132-8.

5. Jamsen E, Sheng P, Halonen P, Lehto MU, Moilanen T, Pajamaki J, Puolakka $T$, Konttinen YT. Spacer prostheses in two-stage revision of infected knee arthroplasty. Int Orthop. 2006;30:257-61.

6. Pietsch M, Hofmann S, Wenisch C. Treatment of deep infection of total knee arthroplasty using a two-stage procedure. Oper Orthop Traumatol. 2006;18:66-87.

7. Freeman MG, Fehring TK, Odum SM, Fehring K, Griffin WL, Mason JB. Functional advantage of articulating versus static spacers in 2-stage revision for total knee arthroplasty infection. J Arthroplast. 2007;22:1116-21.

8. Hsu YC, Cheng HC, Ng TP, Chiu KY. Antibiotic-loaded cement articulating spacer for 2-stage reimplantation in infected total knee arthroplasty: a simple and economic method. J Arthroplasty. 2007;22:1060-6.

9. Park SJ, Song EK, Seon JK, Yoon TR, Park GH. Comparison of static and mobile antibiotic-impregnated cement spacers for the treatment of infected total knee arthroplasty. Int Orthop. 2010;34:1181-6.

10. Chiang ER, Su YP, Chen TH, Chiu FY, Chen WM. Comparison of articulating and static spacers regarding infection with resistant organisms in total knee arthroplasty. Acta Orthop. 2011;82:460-4.

11. Gooding CR, Masri BA, Duncan CP, Greidanus NV, Garbuz DS. Durable infection control and function with the PROSTALAC spacer in two-stage revision for infected knee arthroplasty. Clin Orthop Relat Res. 2011;469:98593.

12. Choi HR, Malchau H, Bedair H. Are prosthetic spacers safe to use in 2-stage treatment for infected total knee arthroplasty? J Arthroplast. 2012;27:14741479.e1471

13. Silvestre A, Almeida F, Renovell P, Morante E, Lopez R. Revision of infected total knee arthroplasty: two-stage reimplantation using an antibioticimpregnated static spacer. Clin Orthop Surg. 2013;5:180-7.

14. Shaikh AA, Ha CW, Park YG, Park YB. Two-stage approach to primary TKA in infected arthritic knees using intraoperatively molded articulating cement spacers. Clin Orthop Relat Res. 2014;472:2201-7.
15. Insall JN, Thompson FM, Brause BD. Two-stage reimplantation for the salvage of infected total knee arthroplasty. 1983. J Bone Joint Surg Am. 1983; 84-A:490.

16. Rosenberg AG, Haas B, Barden R, Marquez D, Landon GC, Galante JO. Salvage of infected total knee arthroplasty. Clin Orthop Relat Res. 1988:29-33.

17. Ding H, Yao J, Chang W, Liu F. Comparison of the efficacy of static versus articular spacers in two-stage revision surgery for the treatment of infection following total knee arthroplasty: a meta-analysis. J Orthop Surg Res. 2017; 12:151.

18. Choi HR, Freiberg AA, Malchau H, Rubash HE, Kwon YM. The fate of unplanned retention of prosthetic articulating spacers for infected total hip and total knee arthroplasty. J Arthroplast. 2014;29:690-3.

19. Gomez MM, Tan TL, Manrique J, Deirmengian GK, Parvizi J. The fate of spacers in the treatment of Periprosthetic joint infection. J Bone Joint Surg Am. 2015;97:1495-502

20. Riley DS, Barber MS, Kienle GS, Aronson JK, von Schoen-Angerer T, Tugwell P, Kiene H, Helfand M, Altman DG, Sox H, Werthmann PG, Moher D, Rison RA, Shamseer L, Koch CA, Sun GH, Hanaway P, Sudak NL, Kaszkin-Bettag M, Carpenter JE, Gagnier JJ. CARE guidelines for case reports: explanation and elaboration document. J Clin Epidemiol. 2017;89:218-35.

21. Ha CW. A technique for intraoperative construction of antibiotic spacers. Clin Orthop Relat Res. 2006;445:204-9.

22. Blumberg HM, Burman WJ, Chaisson RE, Daley CL, Etkind SC, Friedman LN, Fujiwara P, Grzemska M, Hopewell PC, Iseman MD, Jasmer RM, Koppaka V, Menzies RI, O'Brien RJ, Reves RR, Reichman LB, Simone PM, Starke JR, Vernon AA. American Thoracic Society/Centers for Disease Control and Prevention/Infectious Diseases Society of America: treatment of tuberculosis. Am J Respir Crit Care Med. 2003;167:603-62.

23. Willert HG, Bertram H, Buchhorn GH. Osteolysis in alloarthroplasty of the hip The role of bone cement fragmentation. Clin Orthop Relat Res. 1990:108121.

24. Willert HG, Buchhorn GH, Gobel D, Koster G, Schaffner S, Schenk R, Semlitsch M. Wear behavior and histopathology of classic cemented metal on metal hip endoprostheses. Clin Orthop Relat Res. 1996:S160-86.

25. Fink B, Rechtenbach A, Buchner H, Vogt S, Hahn M. Articulating spacers used in two-stage revision of infected hip and knee prostheses abrade with time. Clin Orthop Relat Res. 2011;469:1095-102.

26. Pitto RP, Spika IA. Antibiotic-loaded bone cement spacers in two-stage management of infected total knee arthroplasty. Int Orthop. 2004;28:129-33.

27. Burnett RS, Kelly MA, Hanssen AD, Barrack RL. Technique and timing of twostage exchange for infection in TKA. Clin Orthop Relat Res. 2007;464:164-78.

28. Puri L, Wixson RL, Stern SH, Kohli J, Hendrix RW, Stulberg SD. Use of helical computed tomography for the assessment of acetabular osteolysis after total hip arthroplasty. J Bone Joint Surg Am. 2002;84-a:609-14.

29. Kitamura N, Pappedemos PC, Duffy PR 3rd, Stepniewski AS, Hopper RH Jr, Engh CA Jr, Engh CA. The value of anteroposterior pelvic radiographs for evaluating pelvic osteolysis. Clin Orthop Relat Res. 2006;453:239-45.

30. Horowitz SM, Doty SB, Lane JM, Burstein AH. Studies of the mechanism by which the mechanical failure of polymethylmethacrylate leads to bone resorption. J Bone Joint Surg Am. 1993;75:802-13.

31. Sabokbar A, Fujikawa Y, Murray DW, Athanasou NA. Radio-opaque agents in bone cement increase bone resorption. J Bone Joint Surg Br. 1997;79:129-34

32. Villa T, Carnelli D. Experimental evaluation of the biomechanical performances of a PMMA-based knee spacer. Knee. 2007;14:145-53.

33. Durbhakula SM, Czajka J, Fuchs MD, Uhl RL. Antibiotic-loaded articulating cement spacer in the 2-stage exchange of infected total knee arthroplasty. J Arthroplast. 2004:19:768-74. 